\title{
Light stimulus change evokes an activity response in the rat
}

\author{
BILL P. GODSIL and MICHAEL S. FANSELOW \\ University of California, Los Angeles, California
}

\begin{abstract}
Bright light may be a danger signal for rats because they are more vulnerable to predators in bright environments. We examined the fear-evoking properties of bright light with a novel open-field procedure that confronts a rat with the sudden onset or termination of a bright light gradient. The rats did not freeze but exhibited a transient increase in locomotion to light onset and termination, which we call the activity response (AR). This finding suggests that the $\mathrm{AR}$ is an exploratory response geared at investigating stimulus change. The rats also displayed a preference for dark to the lighting differential that was not due to the novelty or slight heating differential supplied by the lamps. These experiments demonstrate that the sudden onset of bright light engages preencounter defensive behavior, as described by the predatory imminence model (Fanselow \& Lester, 1988). This task is amenable to studying light-evoked defensive responses.
\end{abstract}

According to a functional behavioral systems account of defensive behavior (e.g., Fanselow, 1989), animals have evolved sets of responses that act to solve naturally occurring problems, such as the threat of predation. Predators and other danger signals activate an animal's fear system, which then triggers defensive responses directed at protecting the animal from being killed or injured. This problemoriented view focuses the analysis of fear on how multiple behaviors relate to each other in a coordinated manner to solve specific challenges.

For nocturnal animals, such as the rat, bright light may be an ecologically relevant danger signal. According to this hypothesis, the threat of predation increases as a rat moves into brighter places, because it becomes more visible to predators, and therefore, light is correlated with increased danger. A supportive example can be found in the foraging patterns of nocturnal banner-tail kangaroo rats (Dipdomys spectabilis). These prey animals forage less when the moon illuminates their surface-foraging niche than when the moon does not (Lockard, 1975). These rats are likely in greater danger when the moon is up, because many of their predators use visual cues to hunt.

Consistent with the hypothesis that light signals danger for prey animals, increasing illumination evokes numerous fear behaviors. For instance, lighting differentials evoke a preference for dark, whereby rats tend to avoid brightly illuminated places (e.g., Williams, 1971). Bright illumination evokes thigmotaxis (a tendency to stay near walls; e.g., Valle, 1970), a behavior that is seen in other

This study was supported by Grants NIMH MH62122 to M.S.F. and NRSA MH12402 to B.P.G. The authors thank M. J. Sanders, C. K. Cain, and J. Quinn for their helpful comments on the manuscript. Correspondence should be addressed to M. S. Fanselow, Department of Psychology, University of California, Los Angeles, CA 90095 (e-mail: fanselow@ucla.edu). dangerous situations (Grossen \& Kelley, 1972). Thigmotaxis reduces detectability and the threat of attack from behind and above. Bright light also reduces social interaction (File \& Hyde, 1978), reduces locomotion (e.g., Valle, 1970), often increases defecation (e.g., Roth \& Katz, 1979), and enhances the startle reflex (e.g., Walker \& Davis, 1997a) in the rat. These light-evoked behaviors have been suggested to be indicators of fear or anxiety.

Given its propensity to evoke defensive behaviors, light may influence the expression of other fear behaviors. For example, Fanselow and Lester (1988) noted that "if a lighting differential is present in the immediate vicinity the rat will tend to freeze in the darker area" (p. 192) near a wall. This example demonstrates that light may influence the topography of other defensive behaviors. It also shows how rats may display multiple defensive behaviors together (in this example, freezing, preference for dark, and thigmotaxis). Presumably, their co-expression minimizes danger.

Light-evoked behaviors have been investigated with a variety of procedures. The light/dark box consists of two chambers separated by a wall or corridor. One chamber is dark, and the other brightly lit. With its lighting differential, this task is useful for studying dark-preference behavior (e.g., Whishaw, 1974). The open-field task has been used to compare behavior between different lighting conditions, such as dark, dim, and bright illumination. This task provides a means for studying such behaviors as thigmotaxis, defecation, and locomotion (e.g., Walsh \& Cummins, 1976). Responses to light have also been studied with procedures that provide a stimulus change, such as light onset or light termination. Such procedures have been employed for the study of orienting responses (e.g., Honey, Good, \& Manser, 1998). In addition, Walker and Davis (1997a, 1997b) have demonstrated that an acute exposure to bright light temporarily increases the rat's star- 
tle reflex. This phenomenon suggests that bright light unconditionally evokes low levels of fear or anxiety in the rat and that this fear increases startle magnitude in a manner similar to the enhancement observed in fear-potentiated startle, a learned fear procedure (e.g., Falls \& Davis, 1994).

We wanted to develop a behavioral task amenable to characterizing light-evoked behaviors and their neural substrates. To this end, we developed an open-field procedure in which a rat is placed in a dark open field for $4 \mathrm{~min}$, before it is exposed to a bright light gradient. This task has several features that can aid the study of defensive behavior, including the following: (1) The rats are exposed to what may be an ecologically relevant danger signal, the bright light gradient, (2) the sudden onset of light may simulate or mimic an encounter with a predator, and (3) the rats are exposed to a lighting differential that permits the examination of interactions between the preference for dark and other behaviors.

Predatory imminence theory, a functional behavioral system approach, provides a framework in which to evaluate the fear-evoking properties of bright light in our open-field procedure. This theory specifies a set of rules that predict what defensive behaviors will be displayed under various situations that vary with respect to the level of perceived danger (Fanselow, 1989; Fanselow \& Lester, 1988; Timberlake \& Fanselow, 1994). Animals display preencounter defensive behaviors in situations in which a predator may be present but is undetected and postencounter defensive behaviors when a threat has been detected. In addition, these prey animals display circa-strike defensive behaviors as the attacking predator makes contact with the animal (Fanselow \& Lester, 1988). Freezing is the rat's dominant postencounter defensive behavior (e.g., Fanselow \& Lester, 1988). Activity burst behavior, which is the vigorous unconditional locomotor response to footshock (Fanselow, 1982), as well as escape and fighting (Fanselow \& Sigmundi, 1982), are the rat's dominant circa-strike defensive behaviors.

Given these theoretical constructs, if the sudden onset of a bright gradient in our task evokes a level of fear comparable to that for the detection of a predator, rats should display freezing. If the level of fear is nearer to that in a circa-strike situation, rats should display escape or activity burst behavior. Alternatively, if the sudden onset of the bright gradient evokes a level of fear akin to that in a preencounter defense situation, freezing, escape, fighting, and activity burst behavior should be absent, and risk assessment behaviors, such as stretched-approach, should emerge (Godsil, Tinsley, \& Fanselow, 2003).

Experiments 1 and 2 were performed to characterize the rat's response to the onset and termination of the bright light gradient in a novel open-field task, and the results are discussed within the framework of predatory imminence theory. In Experiment 3, we examined the influence of stimulus novelty on dark preference behavior. Finally, Experiment 4 demonstrates that heat produced by the bright lights does not noticeably influence the behaviors observed in this task.

\section{EXPERIMENT 1}

With our open-field procedure, a rat encounters the sudden onset of a bright illumination gradient after a dark baseline period. This procedure casts a lighting differential across the open-field arena and permits the simultaneous assessments of locomotion and zone preference. Experiment 1 was performed to characterize the rat's behavior during this open-field procedure. Predatory imminence theory predicts that rats should show freezing if the onset of the bright gradient simulates an encounter with a predator (a postencounter defensive situation).

\section{Method}

\section{Subjects}

Twenty-two female Long-Evans rats (200-325 g) were bred and raised at UCLA. The animals had free access to food and water and were kept on a 14:10-h day:night cycle. All the experimental procedures occurred between 6 and $12 \mathrm{~h}$ after light onset.

The subjects were housed in groups until they were transferred to single home cages at the start of the experiment. The experimenter handled the rats twice before the start of testing. Handling consisted of transporting the rats to the area adjacent to the test room and picking up each animal for approximately $10 \mathrm{sec}$.

\section{Open-Field Apparatus}

The open-field arena was a white, translucent polyethylene box (Model CB-80, Iris USA, Pleasant Prairie, WI) with internal dimensions of $69 \mathrm{~cm}$ long $\times 34 \mathrm{~cm}$ wide $\times 30 \mathrm{~cm}$ high. Black electrical tape attached to the underside of the floor marked eight squares $(17 \times 17 \mathrm{~cm})$ that were visible from above. The arena was situated on a table in the center of the room. Three lamps containing single $100-\mathrm{W}$ white light bulbs were positioned at one end of the table (see Figure 1). One lamp was positioned $14 \mathrm{~cm}$ from the center of a short wall of the rectangular arena; one lamp flanked each long side of the arena, $14 \mathrm{~cm}$ from the long walls and $15 \mathrm{~cm}$ from the original short wall. All three lamps were situated $18 \mathrm{~cm}$ above the base of the arena. When lit, the bulbs flooded one end of the open field with light (Zone 1), creating an illumination gradient across the arena. The experimenter could manipulate these lights from the adjacent room, and their luminance output could be adjusted with a dimming switch (Variac, Type W5M, General Radio Company, Concord, MA). A white-noise generator provided background noise (65 dB, A scale). Two cameras (directly adjacent to each other) were suspended from the ceiling directly above the center of the open field. One camera (NightShot setting, Model CCD TRV57, Sony) was used to monitor the open field while the room was dark, and another camera (Cohu, San Diego) was used to monitor the arena while the three lamps illuminated the room. These cameras were connected to a monitor and VCR located in the adjacent room.

\section{Open-Field Testing}

Testing began $20 \mathrm{~min}$ after the animals were transported to the room adjacent to the test room. The rats were tested in two identical open fields that were located in separate rooms. The open-field arenas were cleaned with $5 \%$ sodium hydroxide and rotated $180^{\circ}$ before each test. The rats were tested individually in a sequence counterbalanced for group treatments. The experimenter placed the animal in the center of the open field. The 8-min test began as the door of the test room was closed. At the end of the test, the experimenter returned 


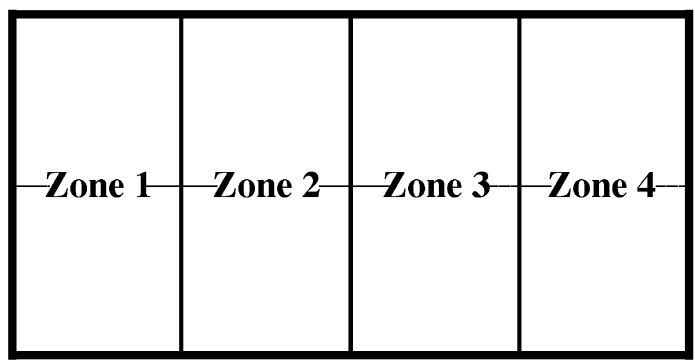

Figure 1. Schematic of the open field showing the position of the lights and the zones used to score zone preference. The middle horizontal line demarcates the squares used to score locomotion.

the subject to its cage and then cleaned the open field in preparation for the next test. For the following experiments, Phase 1 refers to the first $4 \mathrm{~min}$, and Phase 2 refers to the last $4 \mathrm{~min}$ of the test session.

\section{Light Stimuli}

Illumination levels were measured with a light meter (Model 403125, Extech Instruments, Waltham, MA). The light meter's probe sensor was situated in the center of the floor in Zone 1 or Zone 4 (with the tip of the sensor pointed at Zone 1) as the measurements were taken. In the dark condition (Experiments 1, 2, and 4), the open field was $0 \mathrm{fc}$ in Zones 1 and 4 . In the bright gradient condition (Experiments 1, 2, 3, and 4), Zone 1 was approximately $200 \mathrm{fc}$, and Zone 4 was approximately $100 \mathrm{fc}$. In the all-bright condition (Experiment 3 ), the open field was approximately $300 \mathrm{fc}$ in Zone 1 and Zone 4.

\section{Behavioral Measures}

For each behavioral measure, the first author was the observer. $\mathrm{He}$ could not be kept blind to the experimental manipulations because the illumination condition was apparent on the videotape. The experimenter had no a priori hypothesis that might implicitly influence the observations, because the primary purpose of the experiment was the development of a behavioral task and procedure. However, when another observer conducted these behavioral measures, the results were similar (reliability coefficients were above .92 for the locomotion, activity response, and zone preference data).

Locomotion. To provide a measure of locomotion in the open field, an observer counted the number of crossovers a rat performed during each minute of the test. A crossover was defined as a movement from square to square that included all four of the rat's paws. This measure provided an estimate of the distance traveled by each animal.

Activity response. To estimate the rat's change in locomotion in response to a change in illumination, the difference in crossovers between minutes 4 and 5 of the test were calculated [(minute 5 crossover count) - (minute 4 crossover count)].

Zone preference. To estimate the time spent in each zone of the arena, zone preference was scored according to an instantaneous time-sampling procedure with which each animal was observed every $4 \mathrm{sec}$ during the 8 -min test. An observer assigned a position to the rat on the basis of an assessment of where the center of the animal's body was located (excluding the tail). These observations estimated the time that the animals spent in each zone of the arena. A zone consisted of two adjacent squares aligned vertically along the length of the arena. Thus, for this measure, the open field was divided into four equal rectangles $(34 \times 17 \mathrm{~cm}$; Figure 1). Pilot studies had demonstrated that animals spend equal time in Zones 1 and 4 when the open field is dark (approximately 33\%). In addition, rats spend an equal amount of time in Zones 2 and 3 when the arena is dark (approximately 17\%). Thus, animals display a preference for the distal zones of the arena. This pattern reflects the rat's innate thigmotaxic tendency; Zones 1 and 4 flank the arena's corners, where thigmotaxis is normally the most robust. Given these observations, only zone preference data from Zone 4 were analyzed in these experiments.

Freezing. To estimate the percentage of time the rats spent engaging in freezing behavior, freezing was scored according to an instantaneous time-sampling procedure with which each animal was observed every 2 sec during Phase 2 of the test. Freezing was defined as the absence of movement except that required for breathing.

\section{Procedure}

The rats were divided and assigned to two groups (dark/dark [DD] or dark/bright [DB] gradient). For the rats in group DD $(n=11)$, the open field was dark during both Phase 1 and Phase 2 of the 8min test session. For the rats in group DB $(n=11)$, the open field was dark during Phase 1 and had a bright illumination gradient during Phase 2. Locomotion, activity response, zone preference, and freezing measures were scored from videotape after testing.

\section{Statistical Analysis}

In all cases, group differences were considered to be significant when $p<.05$. Except for the cases indicated, an analysis of variance (ANOVA) was used to determine whether there were any group differences. Locomotion and zone preference data for Phase 1 and 
A

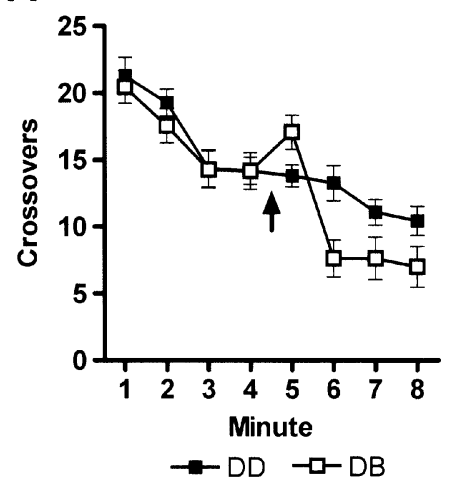

B

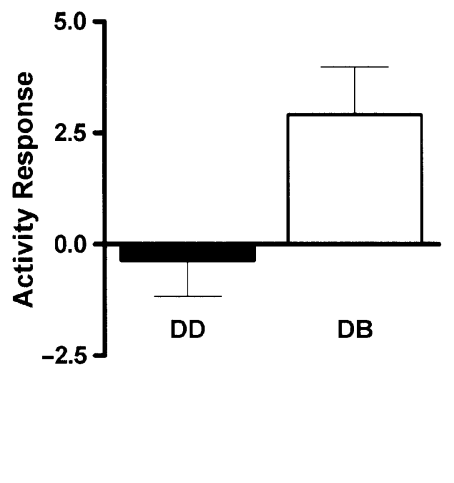

C

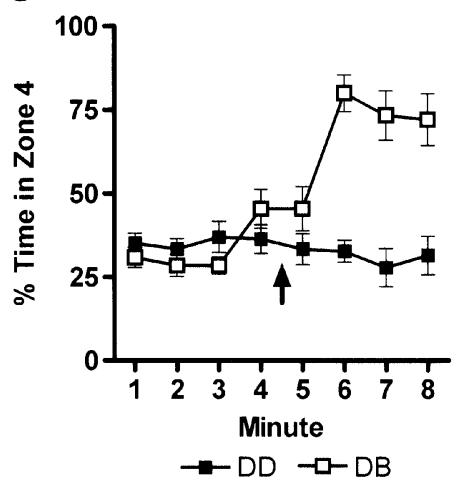

Figure 2. Experiment 1: The sudden onset of the bright gradient evokes an activity response and a preference for dark. (A) Mean locomotion estimated by the number of crossovers counted per minute. (B) Mean activity responses calculated by the change in crossovers between minutes 4 and 5 of testing. (C) Mean zone preference estimated as the percentage of time spent in Zone 4 of the open field during each minute of testing. DD, group dark/dark (black); DB, group dark/bright (white). The arrows shown in panels $\mathrm{A}$ and $\mathrm{C}$ indicate the moment of bright gradient onset for Group DB. Error bars indicate SEMs.

Phase 2 of each experiment were analyzed separately to provide measures separate from a stimulus change; these data were submitted to a repeated measure ANOVA, which included a factor of time (four 1-min blocks).

\section{Results}

Figure 2A contains the locomotion data from Experiment 1. During Phase 1, the locomotion of both groups gradually decreased across the 4-min interval [time, $F(3,60)=21.41, p<.001]$. During Phase 2, group DB displayed more locomotion during minute 5 and less locomotion during the remainder of the session, compared to group DD [minute 5, $F(1,20)=4.67, p<.05$; Phase 2, group $\times$ time interaction, $F(1,60)=5.92, p<.005]$. This pattern demonstrates that during Phase 1 , the rats' locomotion behavior habituated, and the onset of the bright illumination gradient evoked an increase of locomotion.

Figure $2 \mathrm{~B}$ contains the activity response data from Experiment 1. Group DB exhibited an increase in crossovers between minutes 4 and 5 of the test, and group DD displayed similar levels of crossovers during both minutes $[F(1,20)=5.97, p<.05]$. Thus, the stimulus change (dark to bright gradient) increased the locomotion of group DB.

Figure $2 \mathrm{C}$ contains the zone preference data from Experiment 1. Both groups displayed a similar preference for Zone 4 (darkest zone during Phase 2) during Phase 1 $[F(1,60)<1]$. During Phase 2 , group DB spent more time in Zone 4 than did group DD $[F(1,60)=47.94, p<$ $.0001]$. However, these groups spent a similar amount of time in Zone 4 during minute $5[F(1,20)=2.25]$. This pattern of data indicates that the bright gradient triggered a preference for Zone 4 but that the preference was small during the first minute of the bright gradient condition.

Freezing behavior was assessed during Phase 2 of the test. Two out of 11 rats in group DD showed some freezing, and 7 out of 11 showed freezing in group DB. This difference in the distributions was reliable $[U(22)=27$, $p<.05$ ]. However, the overall levels of freezing were very low $(\mathrm{DD}=0.2 \%, \mathrm{DB}=2.7 \%)$. With all but 1 animal, freezing was below $4 \%$ of the time (the higher freezer was in the DB group, and it froze $18 \%$ of the samples). This level of freezing is far less than the freezing that has been shown in experiments in which shock was used.

\section{Discussion}

Rats typically engage in freezing when they encounter a specific threat (see, e.g., Fanselow \& Lester, 1988), but the rats did not display substantial freezing behavior to the bright light gradient ( $<3 \%$ of Phase 2$)$. In contrast, a single mild footshock will evoke substantial levels of freezing, both shortly after shock delivery and during subsequent reexposures to the shock-paired cues (Fanselow, $1982,1990)$. The very low levels of freezing in this task suggests that the sudden onset of a bright illumination gradient does not evoke levels of fear comparable to that in a postencounter defense situation, and it implies that onset of bright light might signal increased danger, but not a specific threat, to the rat (see Table 1).

In addition, many behaviors that resemble circa-strike defensive responses were not prevalent in this experiment. There was no vigorous and sudden activity burst in response to the bright gradient. There were also no audible vocalizations, such as those observed when rats receive moderate intensity footshock (e.g., Goodman, Dyal, Zinser, \& Golub, 1966). Also, we have monitored sound with an ultrasonic detector during our open-field procedure; these unpublished observations suggest that bright gradient onset does not evoke rat ultrasonic vocalizations near the $22-\mathrm{kHz}$ range, which have been suggested to constitute an alarm call in stressful situations (e.g., R. J. Blanchard, Agullana, McGee, Weiss, \& Blanchard, 1992). Given these observations, the sudden onset of a bright gradient does not seem to model either postencounter or 
Table 1

Rat Behaviors on the Predatory Imminence Continuum

\begin{tabular}{|c|c|c|}
\hline $\begin{array}{c}\text { Class of Defensive } \\
\text { Behavior }\end{array}$ & Description & Behaviors \\
\hline Preencounter & $\begin{array}{l}\text { Displayed in situations in which a } \\
\text { predator may be present but the } \\
\text { predator has not yet been } \\
\text { detected }\end{array}$ & $\begin{array}{l}\text { Meal pattern adjustment, dark } \\
\text { preference, }{ }^{*} \text { thigmotaxis, } \\
\text { burying, stretch-approach, } \\
\text { leaving and entering the burrow }\end{array}$ \\
\hline Postencounter & $\begin{array}{l}\text { Displayed when a threat has been } \\
\text { detected in the environment }\end{array}$ & $\begin{array}{l}\text { Freezing, conditional analgesia, } \\
\text { potentiated startle }\end{array}$ \\
\hline Circa-strike & $\begin{array}{l}\text { Displayed when physical contact } \\
\text { with the threat has occurred or } \\
\text { during the time immediately } \\
\text { surrounding such contact }\end{array}$ & $\begin{array}{l}\text { Flight or escape from a threat, } \\
\text { fighting, activity burst behavior }\end{array}$ \\
\hline
\end{tabular}

Note-Adapted from Godsil, Tinsley, and Fanselow (2003). *Rats may engage in this behavior in both preencounter and postencounter defensive situations.

circa-strike defensive situations. Yet it might model a preencounter defensive situation that evokes risk assessment behaviors, such as stretched-approach behavior (R. J. Blanchard \& D. C. Blanchard, 1989).

The rats displayed a transient increase in locomotion during the first minute after light onset. This activity response (AR), which does not resemble the vigorous and sudden activity burst behavior (Fanselow, 1982), could be a milder manifestation of escape behavior, and it is possible that this task simulates a circa-strike situation. Alternatively, the AR could also be an exploratory response. Although the present experimental design does not discriminate between these alternatives, the behaviors expressed during the AR suggest it is not an escape response. The rats did not flee immediately from the bright end of the arena. Instead, they often oriented toward it and then repeatedly approached and retreated from it. The rats appeared vigilant, but they rarely froze. Rearing was common, and it occurred in each zone of the arena. Thus, the rats did not seem to be attempting to find an escape exit, which suggests that the AR is not escape behavior. Instead, the rats seem to vacillate between approach and retreat from the new stimulus in the environment. ${ }^{1}$ In Experiment 2, we attempted to further characterize the AR and examine the possibility that the AR is an escape behavior.

\section{EXPERIMENT 2}

In Experiment 1, the rats displayed a transient increase in locomotion during the minute after bright gradient onset that we call the AR. The second experiment evaluated two possible causes of the AR. First, the response may be an escape behavior elicited by the bright gradient. According to this account, the rat's locomotion increases because it is searching for an escape route or is attempting to move away from the threat. Alternatively, the AR may be an exploratory behavior evoked by the stimulus change, which suggests that the rat's locomotion increases because the stimulus change dishabituates exploration. Experiment 2 was designed to discriminate between these possibilities. We reasoned that if the AR is an escape re- action, it should be elicited only by the onset, but not by the termination, of the bright illumination gradient. This hypothesis assumes that bright light evokes fear and dark does not. In contrast, if the AR is an exploratory behavior, rats should display a locomotion increase whenever there is a stimulus change. To this end, we tested the responses of rats that were presented with the termination of the bright gradient and compared their behavior with that of a bright gradient only control group. We also tested two other groups that replicated those used in Experiment 1. In addition, we conducted a behavioral inventory of the behaviors expressed during minute 5 of the test, to further characterize the activity response.

\section{Method}

The same method as that in Experiment 1 was used in this experiment, except for the changes described below.

\section{Subjects}

Fifty-six female Long-Evans rats bred and raised at UCLA were the subjects.

\section{Behavioral Measures}

Rat behavior was characterized with a behavioral inventory analysis during the 5 th minute of the test (the minute following stimulus change). Five behaviors were scored according to an instantaneous time-sampling procedure. Each animal was observed every $2 \mathrm{sec}$ during minute 5 of the test. These observations yielded an estimate of the percentage of time spent engaging in each behavior. Grooming was defined as licking the paws or using the forepaws to wipe the face or the body. Forward locomotion was defined as movements with all four limbs in a forward direction. Rearing was defined as standing on the rear limbs with the forelimbs off the ground and without movement in any lateral direction. Immobility was defined as the absence of any visible movement except that required for sniffing, whisker movement, and breathing. Stationary activity was defined as any movement of the body that was not grooming, forward locomotion, rearing, sniffing, breathing, or whisker movement. Typically, these behaviors consisted of the following movements: (1) upper body motion that occurred as the hind limbs and the body pivoted near an axis, (2) changes in posture without paw movements, (3) movements of the front paws without movement of the rest of the body, and (4) movement of the head and the neck. When different observers have used this behavioral inventory procedure, they have produced similar results (reliability coefficient $=.87$ ). 


\section{Procedure}

The rats were assigned randomly to one cell of a $2 \times 2$ design that had Phase 1 illumination (two levels: dark vs. bright gradient) and Phase 2 illumination (two levels: dark vs. bright gradient) as factors. The groups were named $\mathrm{DD}, \mathrm{DB}, \mathrm{BD}$, and $\mathrm{BB}(n=14$ per group). During Phase 1, the open field was dark for groups DD and DB and had a bright gradient for groups BD and BB. During Phase 2, the open field was dark for groups DD and BD and had a bright gradient for groups DB and BB. Thus, groups DD and DB were identical to those groups used in Experiment 1. Locomotion, activity response, zone preference, and minute 5 behaviors were scored from videotape after testing.

\section{Results}

Figure 3A contains the locomotion data from Experiment 2 . During Phase 1 , the locomotion of each group gradually decreased across the 4-min interval [time, $F(3,156)=$ $75.91, p<.0001]$. The rats exposed to a dark open field displayed more locomotion than did those exposed to the bright gradient during Phase 1 [Phase 1 illumination, $F(1,156)=52.00, p<.0001]$. This pattern of data demonstrates that the bright gradient suppressed locomotion on the open field, as compared with the dark condition.

The locomotion of the different groups varied with respect to each other during the last 4 min of testing. Averaging across the entire Phase 2 interval, the rats exposed to a dark open field displayed more locomotion than did those exposed to the bright gradient [Phase 2 illumination, $F(1,156)=35.00, p<.0001]$. This pattern demonstrates that the bright gradient suppressed locomotion. Minute 5 locomotion data were submitted to a two-way factorial ANOVA with Phase 1 illumination and Phase 2 illumination as factors. The rats that experienced a stimulus change had more locomotion during minute 5, as compared with those that did not [Phase 1 illumination $\times$ Phase 2 illumination interaction, $F(1,52)=42.21, p<.0001$; Phase 1 il- lumination, $F(1,52)<1$; Phase 2 illumination, $F(1,52)=$ 1.09]. This pattern demonstrates that a stimulus change evoked an AR regardless of the direction of the illumination change (either dark to bright gradient or bright gradient to dark).

As is shown in Figure 3A, groups exposed to the bright gradient during Phase 1 displayed less locomotion during minute 4 than did those exposed to a dark open field. These baseline differences could influence the AR score. Consequently, the ARs for groups DD and DB were analyzed separately from those for groups BB and BD. This analysis compared each stimulus change group with its corresponding Phase 1 control. Each pair was submitted to a one-way ANOVA with Phase 2 illumination as a factor.

Figure $3 \mathrm{~B}$ contains the AR data from Experiment 2. Group DB exhibited an increase in locomotion between minute 4 and 5 of the test, and Group DD displayed a decrease in crossovers between these minutes [Phase 2 illumination, $F(1,26)=7.77, p<.01]$. The onset of the bright gradient evoked an increase in locomotion; this result replicates Experiment 1. Group BD exhibited an increase in locomotion between minutes 4 and 5 of the test, and Group BB displayed similar levels of crossovers during both minutes [Phase 2 illumination, $F(1,26)=11.72, p<$ $.005]$. The termination of the bright gradient evoked an increase in locomotion. Thus, stimulus change evoked an AR for both the dark to bright gradient and the bright gradient to dark open-field sequences.

Figure $3 \mathrm{C}$ contains the zone preference data from Experiment 2. During Phase 1 and Phase 2, groups exposed to bright light spent more time in Zone 4 than did those exposed to a dark open field [Phase 1 illumination, $F(1,156)=$ $49.29, p<.0001$; Phase 2 illumination, $F(1,156)=95.36$, $p<.0001]$. This pattern demonstrates that the bright gradient evoked a preference for dark. In addition, the rats ex-
A

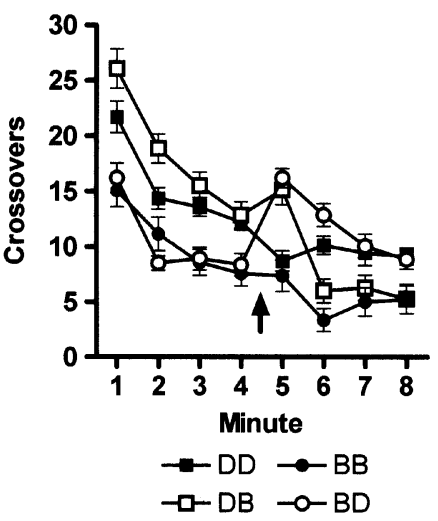

B

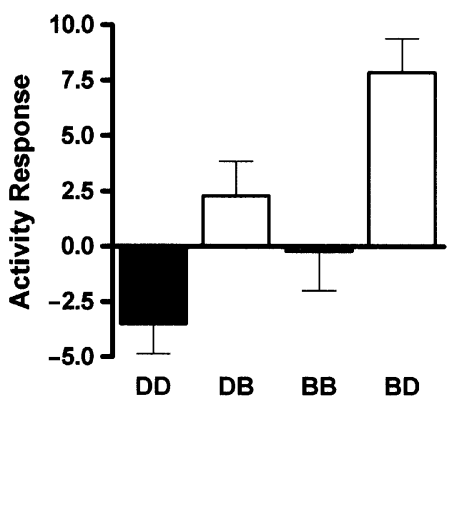

C

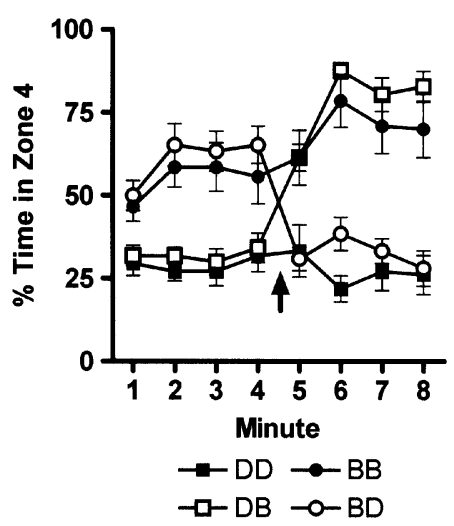

Figure 3. Experiment 2: Bright gradient onset and termination evokes an activity response. (A) Mean locomotion estimated by the number of crossovers counted per minute. (B) Mean activity responses calculated by the change in crossovers between minutes 4 and 5 of testing. (C) Mean zone preference estimated as the percentage of time spent in Zone 4 of the open-field during each minute of testing. DD, group dark/dark (black squares), DB, group dark/bright (white squares), BB, group bright/bright (black circles), BD, group bright/dark (white circles). The arrows shown in panels $A$ and $C$ indicate the moment of stimulus change for Groups DB and BD. Error bars indicate $S E M$ s. 
posed to the bright gradient during Phase 2 (DB and $\mathrm{BB}$ ) appeared to display a greater Zone 4 preference than did the rats exposed to the bright gradient during Phase 1 (BD and $\mathrm{BB}$ ). This pattern suggests that dark-preference behavior is more prevalent when the chamber is more familiar. Thus, exploratory tendencies may compete with the expression of dark preference behavior.

We hypothesized that the AR may be either an exploratory behavior or an escape behavior. Given these possibilities, we may better understand the AR by characterizing the behaviors expressed during minute 5 of testing. To this end, we conducted a behavioral inventory of minute 5 to describe the types of behaviors that rats display after a stimulus change.

Minute 5 behavioral inventory data was submitted to a factorial ANOVA with Phase 1 illumination and Phase 2 illumination as factors. In appropriate cases, these data were also submitted to a one-way ANOVA with treatment (four levels: DD, DB, BD, and BB) as a factor. For these cases, the factorial ANOVA comparisons were not reported.

Table 2 summarizes the behaviors expressed during minute 5 of the test. Group DD engaged in more grooming behavior than did the other groups during minute 5 [treatment, $F(3,52)=2.91, p<.05$ ]. A Fisher's PLSD indicated that group DD groomed more than did groups DB and $\mathrm{BD}$, but not group BB. This pattern suggests that rats are more likely to groom after they have become relatively familiar with the chamber. Rats that experienced a stimulus change engaged in more forward locomotion during minute 5 than did those that did not [Phase 1 illumination $\times$ Phase 2 illumination interaction, $F(1,52)=22.67$, $p<.0001]$. This pattern is consistent with the notion that stimulus change increases forward locomotion. The rats that were exposed to the bright gradient during minute 5 engaged in less rearing than did those exposed to the dark [Phase 2 illumination, $F(1,52)=37.94, p<.0001$ ]. This pattern suggests that the bright gradient suppressed rearing. The rats that were exposed to the bright gradient during minute 5 engaged in more stationary activity than did those exposed to the dark [Phase 2 illumination, $F(1,52)=$ $53.78, p<.0001]$. This pattern suggests that the bright gradient evoked more stationary activity behavior. Groups $\mathrm{DD}, \mathrm{DB}$, and BD displayed negligible levels of immobility during minute 5 of the test; group BB was immobile during about $11 \%$ of minute 5 [treatment, $F(3,52)=3.63$, $p<.05]$. A Fisher's PLSD indicated that group BB dis-

Table 2

Percentages of Time Expressing Each Category of Behavior During Minute 5 of Experiment 2

\begin{tabular}{cccccc}
\hline & \multicolumn{5}{c}{ Behavior Category } \\
\cline { 2 - 6 } Group & Grooming & $\begin{array}{c}\text { Forward } \\
\text { Locomotion }\end{array}$ & Rearing & $\begin{array}{c}\text { Stationary } \\
\text { Activity }\end{array}$ & Immobility \\
\hline DD & 12 & 23 & 44 & 20 & 0 \\
DB & 0 & 36 & 20 & 42 & 1 \\
BD & 1 & 37 & 47 & 15 & 0 \\
BB & 4 & 22 & 24 & 45 & 6 \\
\hline
\end{tabular}

Note-Values are rounded to the nearest integer. D, dark; B, bright. played more immobility than did all the other groups. This result suggests that bright light may evoke immobility, but not immediately after a stimulus change.

\section{Discussion}

Onset and termination of the light gradient caused similar activity responses - namely, the rats displayed an increase in locomotion (as measured by crossovers) to both these changes. This result suggests that the AR is an exploratory behavior and that this task does not represent a circa-strike defensive situation. Presumably, the stimulus change evokes an exploratory response because both light gradient onset and termination alter the environment. Such an interpretation is supported by the types of behaviors expressed during minute 5 of testing. Notably, the stimulus change (either dark to bright gradient or bright gradient to dark) evoked similar levels of forward locomotion. This result is consistent with the interpretation that the AR is an exploratory behavior evoked by a stimulus change in the environment. However, DB animals displayed more stationary activity and less rearing than did BD animals. This pattern demonstrates that, although rats show ARs to stimulus change, the manner in which they explore the arena during the AR is influenced by the presence of the bright gradient.

The rats that were exposed to bright light during minute 5 displayed more stationary activity than did the dark controls. We favor the interpretation that fear causes the increased expression of this behavior, because unpublished findings from our laboratory have demonstrated that previous exposure to footshock enhances the expression of stationary activity in response to light gradient onset (Godsil, Blackmore, \& Fanselow, 2004). Thus, the increased expression of this behavior is correlated with manipulations that likely increase the rat's fear state. That rats show increased stationary activity in the presence of the bright light stimulus, as compared with the dark, suggests that the bright gradient evokes fear or anxiety.

The rats engaged in forward locomotion, stationary activity, and rearing behaviors immediately after the onset of the bright gradient; during this same period, grooming, immobility, and freezing behaviors are absent. The bright gradient suppressed rearing and evoked more stationary activity, as compared with the dark condition. After minute 5, these animals displayed a robust dark preference. This pattern suggests that the onset of the bright gradient seems to evoke two conflicting tendencies: The first is to explore the environment with the AR, and the second is to avoid the bright light.

\section{EXPERIMENT 3}

We have interpreted the results of Experiments 1 and 2 to indicate that bright light evokes a preference for dark. However, the bright light gradient used in this task has a sudden onset, and it is possible that the novelty of the stimulus contributes to the zone preference pattern. Experiment 3 was designed to assess this possibility. Three lamps, 
identical to the originals, were added to the open field at the opposite end from the original fixtures. In addition, rats were tested with a modified procedure that began with an all-bright phase (three lamps at each end of the arena; no lighting differential) followed by a light gradient phase. With this procedure, darkness encroaches upon one end of the arena as the stimulus changes. That is, as the stimulus changes, the all-bright arena becomes dark at one end. This procedure assesses the influence of novelty on the expression of the zone preference. If illumination intensity controls zone preference behavior, the rats should display a dark preference during Phase 2 . This outcome would support the hypothesis that bright light signals danger. Alternatively, if stimulus novelty controls zone preference, rats should avoid the darkest zone of the arena. This outcome would imply that the novel aspects of the light stimulus make a significant contribution to the observed zone preference behavior seen in this task.

\section{Method}

The same method as that in the previous experiments was used in Experiment 3, except for the modifications noted below.

\section{Subjects} jects.

Thirty-two female rats bred and raised at UCLA were the sub-

\section{Open-Field Modification}

Three additional lamps were positioned on the open field at the end opposite the other lamps. As a result, one lamp was positioned $14 \mathrm{~cm}$ from the center of each short wall of the arena; two lamps flanked each long side of the arena, $14 \mathrm{~cm}$ from the long walls, and one of these lamps was $15 \mathrm{~cm}$ from each short wall. Each of these lamps was positioned $18 \mathrm{~cm}$ above the base of the open field, and each set of three lamps could be manipulated from the adjacent room. Thus, during Experiment 3 , six lamps surrounded the open-field arena.

\section{Procedure}

Rats were randomly assigned to two groups (all bright $[\mathrm{AB}]$ or all bright/light gradient [ABL]). Group $\mathrm{AB}(n=16)$ was exposed to the all-bright condition; all six lamps were illuminated during Phases 1 and 2 of the 8-min test. Group ABL $(n=16)$ was exposed to the allbright condition during Phase 1 and the light gradient condition during Phase 2. Locomotion, activity response, zone preference, and freezing measures were scored from videotape after testing.

\section{Results}

Figure 4A contains the locomotion data from Experiment 3. During Phase 1, the locomotion of both groups was similar. During Phase 2, group ABL displayed more locomotion than did group $\mathrm{AB}[F(1,90)=5.74, p<.05]$. Fisher's PLSD post hoc tests revealed that group ABL exhibited more locomotion during minutes 5 and 6 . This pattern of results demonstrates that the onset of the light gradient evoked an increase of locomotion that persisted for 2 min, as compared with controls.

Figure 4B contains the activity response data from Experiment 3. Group ABL exhibited an increase in locomotion between minutes 4 and 5 of the test, and group $\mathrm{AB}$ displayed a decrease in locomotion between these minutes $[F(1,30)=9.63, p<.005]$. Thus, the onset of the light gradient evoked an activity response.

Figure 4C contains the zone preference data from Experiment 3. Both groups displayed a similar preference for Zone 4 during Phase 1 of testing $[F(1,90)=1.20]$. During Phase 2, group ABL spent more time in Zone 4 than did group $\mathrm{AB}[F(1,90)=5.57, p<.05]$. This pattern of data indicates that the light gradient evoked a preference for dark in group ABL.

Freezing behavior was assessed during Phase 2 of the test. Nine out of 14 rats in each group showed some freezing. Again, the overall levels of freezing were low $(\mathrm{AB}=$
A

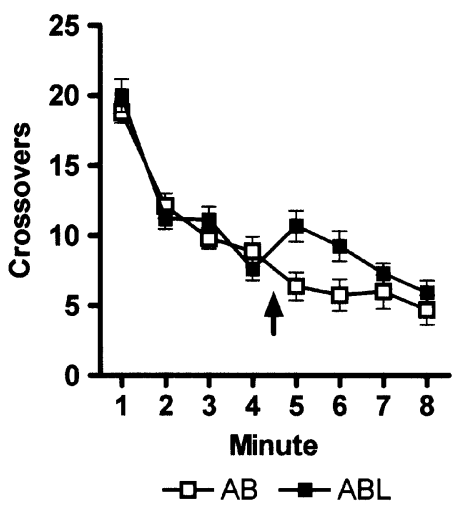

B

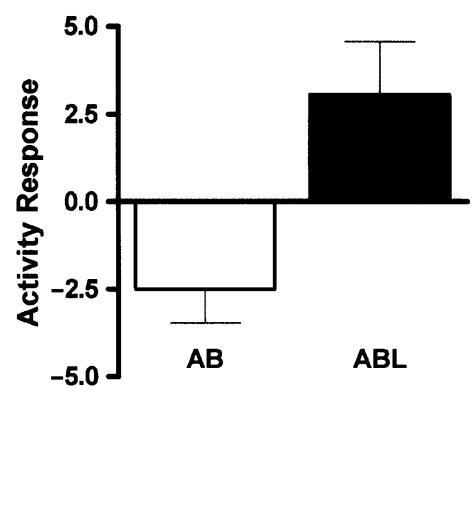

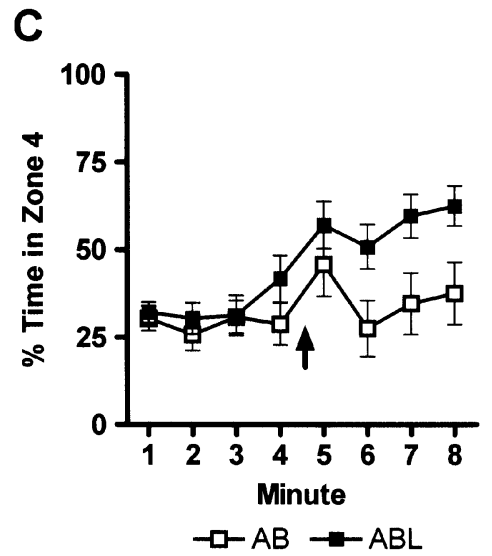

C

Figure 4. Experiment 3: The lighting differential, and not stimulus novelty, evokes a preference for dark. (A) Mean locomotion estimated by the number of crossovers counted per minute. (B) Mean activity responses calculated by the change in crossovers between minutes 4 and 5 of testing. (C) Mean zone preference estimated as the percentage of time spent in Zone 4 of the open field during each minute of testing. AB, group all bright (white), ABL, group all bright/light gradient (black). The arrows shown in panels $A$ and $C$ indicate the moment of bright gradient onset for group $A B L$. Error bars indicate $S E M$ s. 
$5.9 \%, \mathrm{ABB}=2.0 \%)$. This difference in the means was not reliable [group, $F(1,26),=2.56, p=.12$ ]. Two animals from group $\mathrm{AB}$ froze more than $10 \%$ of the session (with freezing scores of $33 \%$ and $14 \%$ ). For the remainder of the animals, freezing was below $10 \%$ of the time. This level of freezing is far less than the freezing found in experiments in which shock was used.

\section{Discussion}

The rats presented with the onset of a light gradient after an all-bright condition displayed an activity response and dark-preference behavior. These results support the interpretation that the bright light evokes the preference for dark, which provides further support for the hypothesis that bright light signals danger. It is unlikely that stimulus change alone evoked the dark-preference behavior (Group BD from Experiment 2 did not display a preference for Zone 4 after the stimulus change).

The magnitude of dark-preference behavior seems to be reduced when compared with that in Experiments 1 and 2. This decrease may indicate that the novelty contributes to the expression of dark-preference behavior (i.e., perhaps the dark preference is reduced because the novelty of dark opposes dark-preference behavior). However, it is also possible that the reduced dark preference is due to a habituation of locomotion processes. Consider group ABL. These rats were presented with an all-bright condition during Phase 1, and they displayed lower locomotion scores that did the groups tested with the dark condition in Experiments 1 and 2. This low level of locomotion is likely the result of the locomotion-suppressing property of bright light, and it contributes to the relative unfamiliarity of the open-field arena. Owing to this unfamiliarity, after the stimulus change, group ABL engaged in locomotion behavior for a longer duration. This increased exploration may contribute to the reduced expression of dark-preference behavior; it seems that the tendency to explore competes with dark-preference behavior. This competition interpretation is supported by the extended duration of the activity response; that is, group ABL displayed an increase in locomotion after the stimulus change that lasted for $2 \mathrm{~min}$ (as opposed to $1 \mathrm{~min}$ ). Thus, it appears that these animals spent more time exploring the arena after the stimulus change. Given these features, it is possible that the novelty of the stimulus contributes to the expression of zone preference behavior but does so to a small extent.

It is worth noting that responses to bright light are known to habituate with repeated exposures to the stimulus. For example, Timberlake, Leffel, and Hoffman (1999) demonstrated that although bright light evokes robust thigmotaxis during the first exposure to the stimulus, as measured by distance traveled away from a wall, this response lessens after repeated trials. This pattern implies that novelty does contribute to the fear-evoking properties of bright light. It might also indicate that, with repeated exposure, rats learn that the maze apparatus is "safe," and therefore, bright light has a tendency to evoke less defensive behavior.

\section{EXPERIMENT 4}

The 100-W incandescent light bulbs used in these experiments emit heat and cause a slight thermal differential on the open field (approximately $3^{\circ} \mathrm{C}$ warmer in Zone 4 than in Zone 1 during the bright phase). This thermal gradient could confound our results. To address this concern, we replicated Experiment 1 and added an additional group whose light source used low-wattage fluorescent bulbs. These bulbs emit light of an intensity similar to that of incandescent bulbs but create a negligible thermal differential $\left(<0.5^{\circ} \mathrm{C}\right)$. If the heating differential caused by the incandescent bulbs influences open-field behaviors, the incandescent and fluorescent bulb groups should display different behavior patterns. If the heating does not influence behavior, these groups should exhibit similar behavior patterns.

\section{Method}

The method used here was identical to that in Experiment 1, except for the modifications described here.

\section{Subjects}

Twenty-seven female rats bred and raised at UCLA were the subjects.

\section{Light Stimuli}

In the bright gradient condition, as before, Zone 1 was approximately $200 \mathrm{fc}$, and Zone 4 was approximately $100 \mathrm{fc}$, for both the fluorescent and the incandescent bulb configurations.

\section{Temperature Measurement}

The temperature of the open-field walls was measured at both the bright and the dark ends of the arena with a digital thermometer (Model 51, Fluke, Everett, WA). The thermometer probe was placed on the internal surface of each short wall (in Zones 1 and 4, at the wall's midpoint, $3 \mathrm{~cm}$ above the arena floor) after the open field had been illuminated with a bright gradient for $4 \mathrm{~min}$.

\section{Procedure}

The rats were randomly assigned to three groups (dark/dark [DD], dark/bright/incandescent [DB/I], and dark/bright/fluorescent $[\mathrm{DB} / \mathrm{F}]$ ). For the rats in group DD $(n=9)$, the open field was dark for both Phase 1 and Phase 2 of the 8 -min test session. For groups DB/I $(n=9)$ and $\mathrm{DB} / \mathrm{F}(n=9)$, the open field was dark during Phase 1 and had the bright gradient condition during Phase 2. For group DB/I, 100-W incandescent bulbs were used in the lamps. For group DB/F, fluorescent $25-\mathrm{W}$ bulbs were used. The light outputs of these bulbs were adjusted with a dimming switch so that the incandescent and the fluorescent bulbs cast light gradients of similar intensities. Locomotion, activity response, and zone preference measures were scored from videotape after testing.

\section{Results}

Figure 5A contains the locomotion data from Experiment 4. During Phase 1, the locomotion of each group gradually decreased across the interval [time, $F(3,72)=45.58$, $p<.0001]$, and the locomotion patterns for each group were similar $[F(2,72)<1]$. Minute 5 locomotion data were submitted to a one-way ANOVA with group as a factor. During Phase 2, groups DB/I and DB/F displayed more locomotion during minute 5 than did group DD $[F(2,24)=15.10, p<.0001]$. Planned comparisons re- 
A

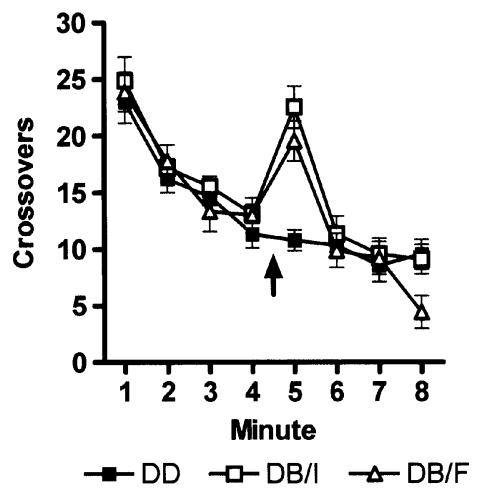

B

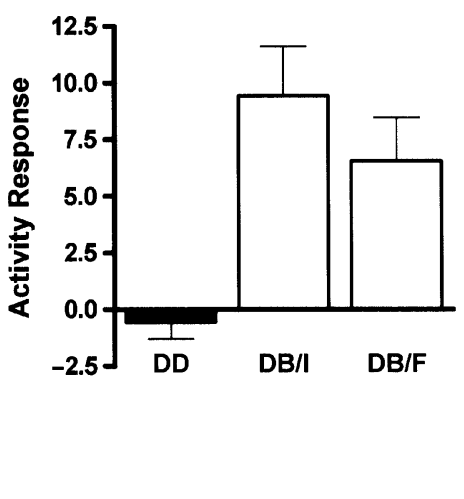

C

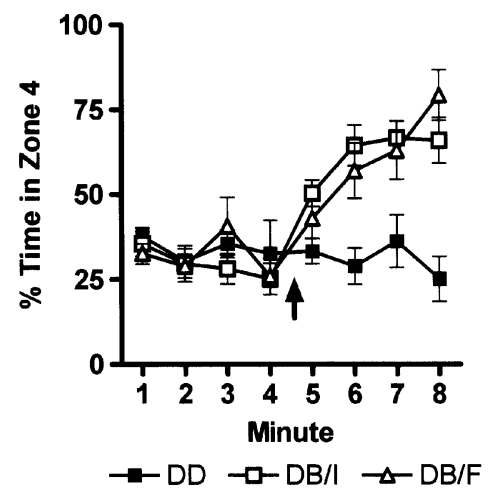

Figure 5. Experiment 4: Heat from the incandescent light bulbs does not influence behavior in this open-field task. (A) Mean locomotion estimated by the number of crossovers counted per minute. (B) Mean activity responses calculated by the change in crossovers between minutes 4 and 5 of testing. (C) Mean zone preference estimated as the percentage of time spent in Zone 4 of the open field during each minute of testing. DD, group dark/dark (black squares), DB/I, group dark/bright/incandescent (white squares), DB/F, group dark/bright/fluorescent (white triangles). The arrows shown in panels $A$ and $C$ indicate the moment of bright gradient onset for Groups DB/I and DB/F. Error bars indicate $S E M$ s.

vealed that both groups $\mathrm{DB} / \mathrm{I}$ and $\mathrm{DB} / \mathrm{F}$ were different from group DD and that they were not different from each other. All three groups had similar locomotion levels during the last $3 \mathrm{~min}$ of the session. This pattern of results demonstrates that the onset of the bright gradient evoked an increase of locomotion for each bulb type group.

Figure 5B contains the activity response data from Experiment 4 . Group DB/I and group DB/F exhibited increases in crossovers between minutes 4 and 5 of the test, and group DD displayed a slight decrease in crossovers across the same interval $[F(2,24)=8.90, p<.005]$. Planned comparisons revealed that both groups $\mathrm{DB} / \mathrm{I}$ and $\mathrm{DB} / \mathrm{F}$ were different from group DD and that they were not different from each other. Thus, the bright gradient evoked an activity response for each bulb type.

Figure 5C contains the zone preference data from Experiment 4. Each group displayed a similar preference for Zone 4 during Phase 1 of the test. During Phase 2, groups $\mathrm{DB} / \mathrm{I}$ and $\mathrm{DB} / \mathrm{F}$ spent more time in Zone 4 than did group $\mathrm{DD}[F(2,72)=19.05, p<.0001]$. Planned comparisons revealed that both groups $\mathrm{DB} / \mathrm{I}$ and $\mathrm{DB} / \mathrm{F}$ were different from group DB and not different from each other. This pattern of data indicates that the bright gradient evoked a preference for dark for both bulb types.

\section{Discussion}

The rats treated with the incandescent and the fluorescent light bulbs displayed similar locomotion, activity response, and zone preference behaviors on the open field. Thus, the slight heating differential created by the incandescent bulbs did not affect the results reported here. Also, the ARs observed in this experiment were appreciably larger than those in the previous experiments.

\section{Additional Analysis}

We have proposed that the bright light gradient evokes a conflict between exploratory and dark-preference behaviors. It is expected that rats that explore more should display less dark-preference behavior. To test this hypothesis, we correlated the activity response data and the mean zone preference data from the last $3 \mathrm{~min}$ of testing for all the animals subjected to the dark/bright gradient treatment (groups DB from Experiments 1 and 2; groups DB/I and $\mathrm{DB} / \mathrm{F}$ from Experiment 4). The last 3 min of testing were used as a sample because these minutes do not overlap with the activity response measure. This comparison revealed a significant negative correlation between these measures $[r(43)=-0.537, p<.0001]$. Thus, the animals with large activity response scores displayed less Zone 4 preference during minutes 6,7 , and 8 of testing. This pattern is consistent with the notion that exploration of stimulus change and the expression of a preference for dark oppose each other in this task.

\section{GENERAL DISCUSSION}

In these experiments, the behavior of rats that are confronted with the sudden onset of a bright gradient in an open field was characterized. The sudden onset of a stimulus presents a rat with both novelty and some degree of danger. Rats express a constellation of behavioral responses in this situation that are generally characterized by a state of conflict between the tendency to explore the novel stimulus and the tendency to avoid the brightly lit location. The stimulus change evokes exploratory behavior, and the bright illumination differential triggers dark-preference behavior and increased stationary activity behavior. 
The present results support the hypothesis that the sudden onset of the bright gradient evokes low levels of fear. Intense fear typically arrests exploration (e.g., freezing) or provokes vigorous and sudden bouts of movement (e.g., escape or activity burst behavior). These behaviors were not observed. Instead, the onset of the bright gradient seems to elicit exploratory behavior. Moreover, just after the onset of the bright gradient, rearing behavior seems to be supplanted by stationary activity behavior. Like rearing, these behaviors might provide a means to sample the environment. However, stationary activity appears subjectively more cautious because this behavior is composed of body postures that keep rats low to the ground (as compared with rearing). Indeed, some forms of stationary activity resemble stretch-approach behavior that has been classified as a fear behavior (e.g., R. J. Blanchard \& D. C. Blanchard, 1989). Thus, stationary activity behavior might provide risk assessment in situations that present slight levels of risk or predatory imminence, such as the bright gradient condition.

In our task, rats display a robust preference for dark after they have explored the stimulus change on the open field. Thus, rats display dark-preference behavior after they have had the opportunity to determine that no threats are present on the open field. This pattern of responding supports the interpretation that our task engages preencounter defensive behaviors, which emerge in situations that maintain a level of risk but a specific threat is absent (Fanselow \& Lester, 1988).

Moreover, according to predatory imminence theory, rats normally engage in a preferred activity pattern that may be perturbed by various threats (Fanselow \& Lester, 1988). Importantly, "pre-encounter defense is not a response to a specific predatory individual ... the prey alters its activity patterns according to the likelihood of an encounter [with a threat] while engaged in a particular activity" (Fanselow \& Lester, 1988, p. 188). Within this theoretical schema in this open-field task, the dark condition more closely approximates the preferred activity pattern, and onset of a bright light moves the rat into a preencounter defensive mode. In other words, light is an ecologically relevant danger signal, and the preference for dark is a preencounter defensive response that is evoked by the resulting lighting differential.

D. C. Blanchard, R. J. Blanchard, Lee, and Fukunaga (1977) reported that rats display some immobility when they are exposed to a bright light in an open field. Our results indicate that the bright gradient does not evoke much freezing or immobility in the rat. It is conceivable that rats do not display immobility in our task because the bright gradient permits rats to avoid the aversive, fear-provoking light stimulus. The ability to avoid the light may reduce the rat's level of perceived danger. The freezing results in Experiment 3 provide some support for this hypothesis. Although not statistically reliable, the rats that were exposed to bright light without a gradient tended to show more freezing than did the rats that were exposed to bright gradient during Phase 2. Yet these levels of freezing were far lower than those reported by D. C. Blanchard et al. (1977).

Our open-field procedure can be used to investigate the neural substrates of defensive reactions to bright light. For example, Walker and Davis (1997a) suggested that a rat becomes anxious when it is exposed to bright light and that, as a result, the animal displays an increased magnitude of the startle reflex. Walker and Davis (1997b) reported that chemical inactivation of the bed nucleus of the stria terminalis or the basolateral nucleus of the amygdala disrupted this light-potentiated startle phenomenon. Our task can be used to test the generality of the involvement of these brain regions in the expression of light-evoked fear behaviors.

The bulk of research relevant to the predatory imminence theory has focused on postencounter defensive responses, such as freezing, and circa-strike defensive responses, such as the activity burst. Indeed, within this framework, the only systematic analysis of preencounter behavior has been provided by experiments that study shifts in meal pattern behavior in response to changes in shock density (Fanselow, Lester, \& Helmstetter, 1988; Helmstetter \& Fanselow, 1993). By investigating responses to the onset of bright light, the aim of the present experiments was to further characterize preencounter defense behaviors. Notably, preencounter defensive behaviors may have relevance to human anxiety. For example, chronically anxious humans spend a sizable amount of time adjusting their preferred activity patterns to reduce anxiety (e.g., Bouton et al., 2001; Robins \& Regier, 1991). Our procedure may represent an animal model for these types of human behaviors.

\section{REFERENCES}

Blanchard, D. C., Blanchard, R. J., Lee, E. M. C., \& Fukunaga, K. K. (1977). Movement arrest and the hippocampus. Physiological Psychology, 5, 331-335.

Blanchard, R. J., Agullana, R., McGee, L., Weiss, S., \& BlanCHARD, D. C. (1992). Sex differences in the incidence and sonographic characteristics of antipredator ultrasonic cries in the laboratory rat (Rattus norvegicus). Journal of Comparative Psychology, 106 270-277.

BLANCHARD, R. J., \& BLANCHARD, D. C. (1989). Antipredator defensive behaviors in a visible burrow system. Journal of Comparative Psychology, 103, 70-82.

Bouton, M. E., MineKa, S., \& Barlow, D. H. (2001). A modern learning theory perspective on the etiology of panic disorder. Psychological Review, 108, 4-32.

FALLS, W. A., \& DAVIS, M. (1994). Fear-potentiated startle using three conditioned stimulus modalities. Animal Learning \& Behavior, 22, 379-383.

FAnselow, M. S. (1982). The postshock activity burst. Animal Learning \& Behavior, 10, 448-454.

FANSELOW, M. S. (1989). The adaptive function of conditioned defensive behavior: An ecological approach to Pavlovian stimulus-substitution theory. In R. J. Blanchard \& P. F. Brain (Eds.), Ethoexperimental approaches to the study of behavior (pp. 151-166). Norwell, MA: Kluwer.

FANSELOW, M. S. (1990). Factors governing one-trial contextual conditioning. Animal Learning \& Behavior, 18, 264-270.

FANSElow, M. S., \& LeSTER, L. (1988). A functional behavioristic ap- 
proach to aversively motivated behavior: Predatory imminence as a determinant of the topography of defensive behavior. In R. C. Bolles \& M. D. Beecher (Eds.), Evolution and learning (pp. 185-211). Hillsdale, NJ: Erlbaum.

Fanselow, M. S., Lester, L. S., \& Helmstetter, F. J. (1988). Changes in feeding and foraging patterns as an antipredator defensive strategy: A laboratory simulation using aversive stimulation in a closed economy. Journal of the Experimental Analysis of Behavior, 50, 361-374.

FANSELOW, M. S., \& SigmUNDI, R. A. (1982). The enhancement and reduction of defensive fighting by naloxone pretreatment. Physiological Psychology, 10, 313-316.

FILE, S. E., \& HYDE, J. R. (1978). Can social interaction be used to measure anxiety? British Journal of Pharmacology, 62, 19-24.

Godsil, B. P., BLackmore, M. A., \& Fanselow, M. S. (2004). Modulation of an activity response with associative and nonassociative fear in the rat. Manuscript submitted for publication.

Godsil, B. P., Tinsley, M., \& Fanselow, M. S. (2003). Motivation. In I. B. Weiner (Series Ed.) \& A. F. Healy \& R. W. Proctor (Vol. Eds.), Handbook of psychology: Vol. 4. Experimental psychology (pp. 3360). New York: Wiley.

Goodman, E. D., Dyal, J. A., Zinser, O., \& Golub, A. (1966). UCR morphology and shock intensity. Psychonomic Science, 5, 431-432.

Grossen, N. E., \& Kelley, M. J. (1972). Species-specific behavior and acquisition of avoidance behavior in rats. Journal of Comparative \& Physiological Psychology, 81, 307-310.

HelmstetTer, F. J., \& FANSELOW, M. S. (1993). Aversively motivated changes in meal patterns of rats in a closed economy: The effects of shock density. Animal Learning \& Behavior, 21, 168-175.

Honey, R. C., Good, M., \& MANSER, K. L. (1998). Negative priming in associative learning: Evidence from a serial-habituation procedure. Journal of Experimental Psychology: Animal Behavior Processes, 24, 229-237.

LOCKARD, R. B. (1975). Experimental inhibition of activity of kangaroo rats in the natural habitat by an artificial moon. Journal of Comparative \& Physiological Psychology, 89, 263-266.
Robins, L. N., \& Regier, D. A. (1991). Psychiatric disorders in America: The epidemiologic catchment area study. New York: Free Press. Roth, K. A., \& KaTZ, R. J. (1979). Stress, behavioral arousal, and openfield activity: A reexamination of emotionality in the rat. Neuroscience \& Biobehavioral Reviews, 3, 247-263.

TIMBERLAKE, W., \& FANSELOW, M. S. (1994). Introduction to symposium on behavior systems: Learning, neurophysiology, and development. Psychonomic Bulletin \& Review, 1, 403-404.

Timberlake, W., LefFel, J., \& Hoffman, C. M. (1999). Stimulus control and function of arm and wall travel by rats on a radial arm floor maze. Animal Learning \& Behavior, 27, 445-460.

VALLE, F. P. (1970). Effects of strain, sex, and illumination on open-field behavior of rats. American Journal of Psychology, 83, 103-111.

WALKER, D. L., \& DAVIS, M. (1997a). Anxiogenic effects of high illumination levels assessed with the acoustic startle response in rats. $\underline{B-}$ ological Psychiatry, 42, 461-471.

WALKER, D. L., \& DAVIS, M. (1997b). Double dissociation between the involvement of the bed nucleus of the stria terminalis and the central nucleus of the amygdala in startle increases produced by conditioned versus unconditioned fear. Journal of Neuroscience, 17, 9375-9383.

Walsh, R. N., \& Cummins, R. A. (1976). The open-field test: A critical review. Psychological Bulletin, 83, 482-504.

WHISHAW, I. Q. (1974). Light avoidance in normal rats and rats with primary visual system lesions. Physiological Psychology, 2, 143-147.

Williams, D. I. (1971). Maze exploration in the rat under different levels of illumination. Animal Behaviour, 19, 365-367.

\section{NOTE}

1. A sample video clip, which shows a rat exploring the open field immediately before and after the onset of the bright illumination gradient, can be viewed at http://www.psych.ucla.edu/fanselowlab/ar.html.

(Manuscript received November 26, 2003 revision accepted for publication April 2, 2004.) 